\title{
Implications of the HST/FGS parallax of SS Cygni on the disc instability model
}

\author{
M. R. Schreiber ${ }^{1,2}$ and B. T. Gänsicke ${ }^{1}$ \\ 1 Universitäts-Sternwarte, Geismarlandstr. 11, 37083 Göttingen, Germany \\ 2 Department of Astronomy, University of Cape Town, Private Bag, Rondebosch 7700, Cape Town, South Africa
}

Received 2 November 2001 / Accepted 6 November 2001

\begin{abstract}
We analyse the consequences of the recently measured parallax of SS Cygni (Harrison et al. 1999) on the accretion disc limit cycle model. Using the observed long term light curve of SS Cyg and $d=166 \pm 12 \mathrm{pc}$, we obtain for the mean mass transfer rate $\bar{M}_{\mathrm{tr}}=4.2 \pm 1.7 \times 10^{17} \mathrm{~g} \mathrm{~s}^{-1}$. In addition, we calculate the vertical structure of the accretion disc taking into account heating of the outer disc by the stream impact. Comparing the mean accretion rate derived from the observations with the calculated critical mass transfer rate, we find that the disc instability model disagrees with the observed long term light curve of SS Cyg as $\overline{\dot{M}}_{\text {tr }}$ is greater or similar to the critical mass transfer rate. The failure of the model indicated by this result can be confirmed by considering that the accretion rate at the onset of the decline should be exactly equal to the value critical for stability. In contrast to this prediction of the model, we find that the accretion rate required to explain the observed visual magnitude at the onset of the decline must be significantly higher than the critical mass transfer rate. Our results strongly suggest that either the usually assumed temperature dependence of the viscosity parameter $\alpha$ is not a realistic description of the disc viscosity, that the mass transfer rate in SS Cyg noticeably increases during the outbursts or, finally, that the HST distance of $166 \pm 12 \mathrm{pc}$, is too high.
\end{abstract}

Key words. accretion, accretion discs - binaries: close - stars: individual: SS Cygni - novae, cataclysmic variables

\section{Introduction}

In non-magnetic cataclysmic variables (CVs) (Warner 1995, for an encyclopaedic review) a white dwarf accretes material from the Roche-lobe filling secondary via an accretion disc. In many CVs, sudden brightenings - called dwarf nova outbursts - are observed. These eruptions are generally thought to result from thermal instabilities in the accretion discs (the disc instability model (DIM), see e.g. Cannizzo 1993b; Ludwig et al. 1994, for parameter studies). Calculations of the steady-state solution of the vertical disc structure for a given radius show a hysteresis relation in the surface-density vs. accretion rate plane - the so-called "S-curve". In principle, a limit cycle behaviour can be maintained by a mean mass-transfer rate which is between the critical rate needed to stay in a cold quiescent state and that needed to remain in the hot outburst state. However, the obtained light curves using a constant viscosity parameter $\alpha$ show only low amplitude outbursts (Smak 1984). In order to match the observed outburst light curves, it is necessary to assume a drastic change of the viscosity parameter $\alpha$ between the hot and

Send offprint requests to: M. R. Schreiber, e-mail: mschrei@uni-sw.gwdg.de the cold state, $\alpha_{\text {hot }}$ and $\alpha_{\text {cold }}$, with $\alpha_{\text {hot }} / \alpha_{\text {cold }}$ typically $5-10$ but with unexplained exceptions $\alpha_{\text {hot }} / \alpha_{\text {cold }} \sim 10^{3}$ e.g. for WZSge. Although the change of $\alpha$ is a somewhat arbitrary assumption, the DIM has become the generally accepted explanation of dwarf nova outbursts as it agrees with observational and theoretical constraints (see Cannizzo 1993b). Recently, additional effects such as disc radius variations (Hameury et al. 1998), irradiation (Hameury et al. 1999; Schreiber et al. 2000a; Schreiber \& Gänsicke 2001), mass transfer variations (Schreiber et al. 2000b; Buat-Ménard et al. 2001) and the effects of the accretion stream impact (Schreiber \& Hessman 1998; Buat-Ménard et al. 2001; Stehle et al. 2001) have been included in the model.

\section{The absolute brightest dwarf nova: SS Cyg}

The dwarf nova SS Cyg is among the visually brightest CVs and has a detailed long term light curve covering more than a century. SS Cyg has, therefore, become the reference object for accretion disc instability analyses, and its observed light curve has been used to calibrate the viscosity parameter $\alpha$. Cannizzo \& Mattei (1992) examined the long term light curve of SS Cyg (Fig. 1) in detail in 


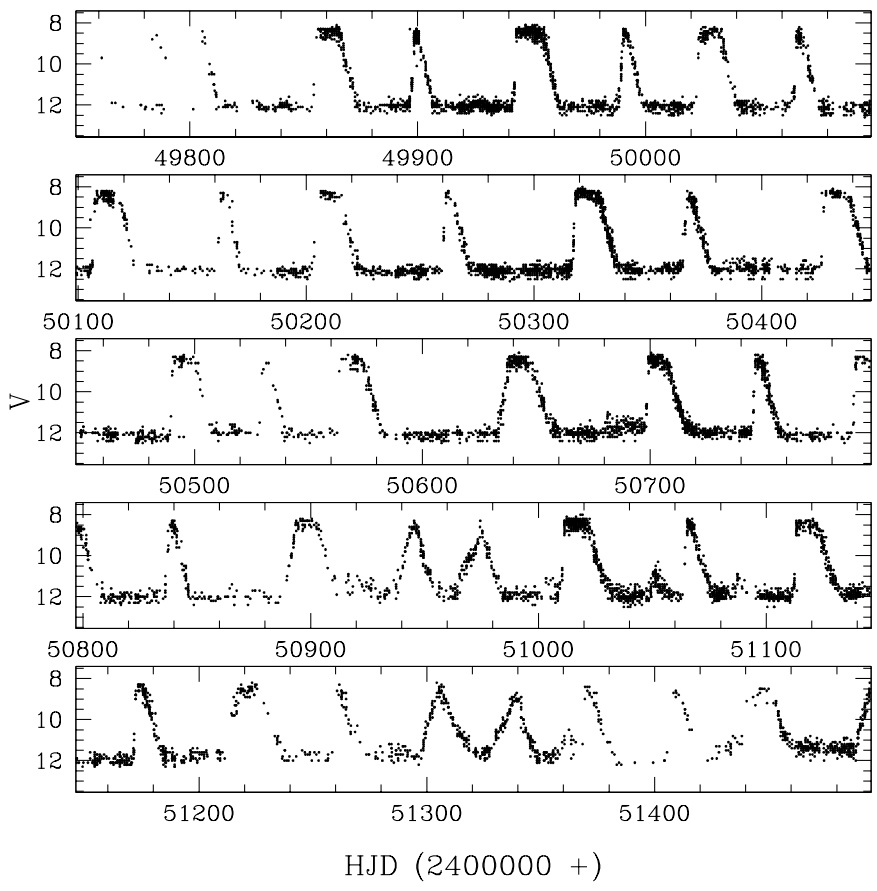

Fig. 1. The visual long term light curve of SSCyg compiled from observations made by members of the Association Francaise des Observateurs d'Étoiles Variables.

order to test the limit cycle model (Cannizzo 1993a) and found a mean outburst duration of $t_{\text {out }}=10.76 \mathrm{~d}$ and a mean quiescence time of $t_{\text {qui }}=38.71 \mathrm{~d}$ (corresponding to a mean cycle duration of $t_{\text {cyc }}=49.47 \mathrm{~d}$ ). In a later paper, Cannizzo \& Mattei (1998) again examined the light curve of SSCyg, deriving the rise and decay times. Only the mean values are important in the context of our analysis: $t_{\text {ris }}=0.5 \mathrm{~d}, t_{\mathrm{dec}}=2.5 \mathrm{~d}$. These values are obtained using a conservatively low estimate of the outburst magnitude of $m_{v}=8.5$.

However, even though the brightness variation of SS Cyg has been monitored to a high level of precision, its distance has been highly uncertain. As a consequence, the absolute magnitude and the accretion rate, have also been ill-defined. Recently, a high-quality parallax of SS Cyg was obtained using the Hubble Space Telescope (HST) Fine Guidance Sensor (FGS), which moves the system to a distance 1.5-2 times larger than previously thought (Harrison et al. 1999). The HST/FGS distance of $166 \pm 12 \mathrm{pc}$ is confirmed by Beuermann (2000), who showed that the accretion disc in SS Cyg contributes significantly to the observed $K$ band flux. As a consequence of the now firmly established (larger) distance, the absolute magnitude of SS Cyg in outburst increased, too. Figure 2 shows the absolute visual magnitudes of dwarf novae at outburst maximum as a function of their orbital period, adapted from Warner (1987). The arrow displays the impact of the HST/FGS parallax on SS Cyg: the system is now the absolute brightest dwarf nova in outburst and its maximum absolute magnitude is brighter than one would expect from its orbital period (Cannizzo 1998).

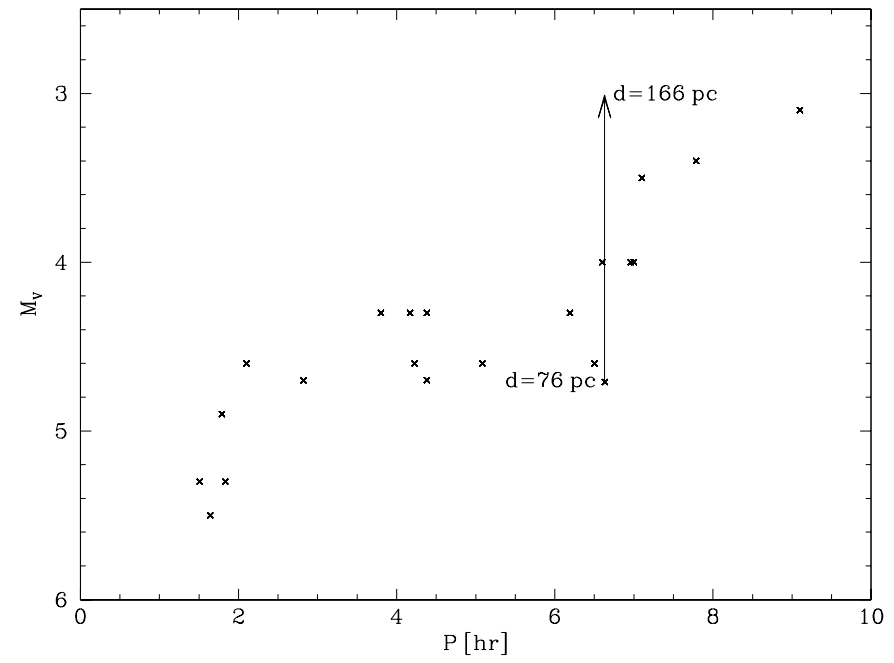

Fig. 2. The absolute magnitudes of dwarf novae adapted from Warner (1987) corrected to a mean inclination of $i=56.7$ and with $m_{v}=8.5$ for the maximum magnitude of SSCyg. Due to the increased distance, SS Cyg has become the absolute brightest dwarf nova.

The mean accretion rate in SS Cyg inferred from its long term light curve depends, in addition to the absolute magnitude, on the inclination $i$ of the accretion disc. This system parameter is somewhat uncertain, most studies of SS Cyg suggest $i=37^{\circ} \pm 5^{\circ}$ (Ritter \& Kolb 1998). A significantly higher value of $i \simeq 57^{\circ}$ was derived by Voloshina \& Khruzina (1997), but, throughout this paper we assume the lower value. To account for the influence of the inclination on the absolute magnitude we follow Paczynski \& Schwarzenberg-Czerny (1980):

$\Delta M_{v}(i)=-2.5 \log \left[\frac{(0.4+0.6 \cos i) \cos i}{0.4}\right]$,

where we used a limb darkening coefficient $u=0.6$. Notice, Eq. (1) gives the inclination correction corresponding to a mean inclination $i=56.7$ (Fig. 2).

Throughout this paper we use the system parameters of SS Cyg given in the literature, i.e. the primary mass $M_{\mathrm{wd}}=1.19 \pm 0.05 M_{\odot}($ Ritter \& Kolb 1998; van Teeseling $1997)$, secondary mass $M_{\mathrm{sec}}=0.70 M_{\odot}$, and orbital period in hours $P_{\mathrm{hr}}=6.6$ (Ritter \& Kolb 1998).

\section{Deriving the critical and the mean mass transfer rate}

The goal of this section is to check if the "standard" disc instability model can reproduce the absolute magnitude of the outbursts of SS Cyg, taking into account the larger HST/FGS distance, the inclination, and the mean outburst time scales derived from the long term light curve.

\subsection{The mean mass transfer rate}

Figure 3 shows a light curve computed with the FE/FD DIM code used for the first time in Schreiber \& Hessman (1998) and described in detail in Schreiber et al. (2000b). 

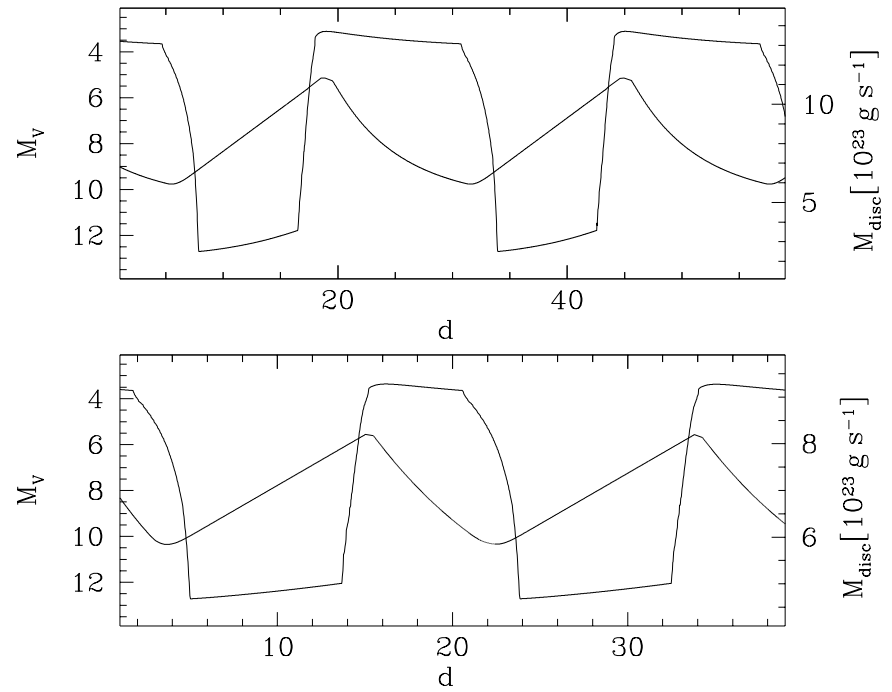

Fig. 3. Simulated light curves using the orbital parameters of SS Cyg, $\alpha_{\mathrm{h}}=0.5, \alpha_{\mathrm{c}}=0.05, \log \left(\dot{M}_{\mathrm{tr}}\right)=17.7$ (upper panel) and $\log \left(\dot{M}_{\mathrm{tr}}\right)=17.4$.

We adapted the parameters suggested by Ludwig et al. (1994) and two different values for the mass transfer rate.

During an outburst cycle the disc subsequently goes through a phase of quiescence, rise, adjustment, quasistationary outburst state, and decay. After the heating front has brought the entire disc into the hot state (rise), the disc redistributes its mass (adjustment) until it accretes without radial variations of the accretion rate (quasi-stationary state). Almost all the mass accreted during an outburst cycle is transferred onto the white dwarf during this quasi-stationary outburst state (Fig. 3). Thus, to derive a lower limit for the mass accreted during a mean outburst cycle, one simply has to multiply the accretion rate of a stationary standard disc which reaches $m_{V}=8.5 \pm 0.1$ with the mean quasi-steady state duration, $t_{\mathrm{qs}} \sim 5.76 \mathrm{~d}^{1}$. Throughout this paper we assume a very large disc for SS Cyg during outburst, i.e. $(90 \pm 5) \%$ of the primary's Roche radius $R_{\mathrm{L} 1}$. (Ritter 1980; Harrop-Allin \& Warner 1996) $\left(0.9 R_{\mathrm{L} 1}=5.83 \times 10^{10} \mathrm{~cm}\right.$ for the binary parameter of SS Cyg). Following Buat-Ménard et al. (2001), we describe the additional heating of the outer disc due to the stream disc impact with

$Q_{\mathrm{i}}=\eta_{\mathrm{i}} \frac{G M_{\mathrm{wd}} \dot{M}_{\mathrm{tr}}}{2 R_{\mathrm{d}}} \frac{1}{2 \pi R_{\mathrm{d}} \Delta R_{\mathrm{hs}}} \exp \left(\frac{R_{\mathrm{d}}-R}{\Delta R_{\mathrm{hs}}}\right)$,

where $R_{\mathrm{d}}$ is the outer disc radius and $\eta_{\mathrm{i}}$ the efficiency of disc heating. In this section we assume $\eta_{\mathrm{i}}=1.0 \pm 0.25$ and the Keplerian kinetic energy being released over a radial extent of $\Delta R_{\mathrm{hs}}=0.1 R_{\mathrm{d}}$ with an exponential attenuation (Buat-Ménard et al. 2001). In addition, we include irradiation of the disc by the white dwarf and the boundary layer using Eqs. (4) and (5) of Schreiber et al. (2000b) with an albedo of the accretion disc of $\beta=0.75 \pm 0.25$. To account for the contribution of the irradiated secondary (K4/5) we

\footnotetext{
1 Assuming an adjustment time of $t_{\text {ad }} \sim 2 \mathrm{~d}$.
}

Table 1. Assumed system parameter.

\begin{tabular}{|c|c|}
\hline Binary parameter & Outburst parameter \\
\hline$M_{\mathrm{wd}}=1.19 \pm 0.05 M_{\odot}$ & $t_{\text {out }}=10.76 \mathrm{~d}$ \\
\hline$M_{\mathrm{sec}}=0.70 M_{\odot}$ & $t_{\text {qui }}=38.71 \mathrm{~d}$ \\
\hline$=37^{\circ} \pm 5^{\circ}$ & $t_{\mathrm{cyc}}=49.47 \mathrm{~d}$ \\
\hline$=6.6 \mathrm{hr}$ & $t_{\text {ris }}=0.5 \mathrm{~d}$ \\
\hline$=166 \pm 12 \mathrm{pc}$ & $t_{\mathrm{dec}}=2.5 \mathrm{~d}$ \\
\hline$R_{\mathrm{d}} \quad=0.90 \pm 0.05 R_{L 1}$ & $m_{V}=8.5 \pm 0.1$ \\
\hline \multicolumn{2}{|c|}{ Stream impact and irradiation heating efficiencies } \\
\hline$\eta_{\mathrm{i}}=1.0 \pm 0.25$ & $(1-\beta)=0.25 \pm 0.25$ \\
\hline \multicolumn{2}{|c|}{ Contribution of the irradiated secondary } \\
\hline \multicolumn{2}{|c|}{$m_{V, \mathrm{sec}} \geq 11$} \\
\hline
\end{tabular}

used the code BINARY ++ written by A. van Teeseling (1998) and found even for an extremely high accretion rate of $\dot{M}_{\text {out }}=4 \times 10^{18} \mathrm{~g} \mathrm{~s}^{-1}$ the visual magnitude of the irradiated secondary being $m_{\mathrm{v}, \mathrm{sec}} \geq 11$. We consider $m_{\mathrm{v}, \mathrm{sec}}=11$ as an upper limit for the contribution of the irradiated secondary throughout this paper.

The accretion rate necessary to reproduce the observed visual flux during the outburst can be described with a power-law:

$$
\begin{aligned}
\dot{M}_{\text {out }}= & 3.62 \times 10^{18} \mathrm{~g} \mathrm{~s}^{-1}\left(\frac{d}{166 \mathrm{pc}}\right)^{3.990}\left(\frac{i}{37^{\circ}}\right)^{1.499} \\
& \times\left(\frac{M_{\mathrm{wd}}}{1.19 M_{\odot}}\right)^{-1.449}\left(\frac{\eta_{\mathrm{i}}}{1.0}\right)^{-0.023}\left(\frac{R_{10}}{5.83}\right)^{-1.130} \\
& \times\left(\frac{\beta}{0.75}\right)^{0.058}\left(\frac{m_{V}}{8.5}\right)^{-15.508}
\end{aligned}
$$

With the parameters and their uncertainties given in Table 1, this results in

$\dot{M}_{\text {out }}=(3.62 \pm 1.47) \times 10^{18} \mathrm{~g} \mathrm{~s}^{-1}$.

The accreted mass has to be transferred to the disc during a mean outburst cycle by a mean mass transfer rate:

$\overline{\dot{M}}_{\mathrm{tr}} \sim \frac{\dot{M}_{\mathrm{out}} t_{\mathrm{qs}}}{t_{\mathrm{cyc}}}=(4.2 \pm 1.7) \times 10^{17} \mathrm{~g} \mathrm{~s}^{-1}$.

This value has to be compared with the critical mass transfer rate.

\subsection{The critical mass transfer rate}

In the absence of stream impact heating the critical accretion rate is given by the accretion rate at the upper turning point of the S-curve $\dot{M}_{\mathrm{A}}$ and can be approximated by a power-law, i.e.

$\dot{M}_{\mathrm{A}}=\dot{M}_{\text {crit }}=9.5 \times 10^{15} \mathrm{~g} \mathrm{~s}^{-1} R_{10}^{2.64} M_{\mathrm{wd}}{ }^{-0.88}$, 
where $R_{10}=R / 10^{10} \mathrm{~cm}$ (see e.g. Schreiber \& Gänsicke 2001; Hameury et al. 1998; Ludwig et al. 1994) ${ }^{2}$.

Taking into account heating of the outer edge of the disc by the impact of the stream, the critical mass transfer rate is given by an implicit equation as the turning point of the modified S-curve $\dot{M}_{\mathrm{A}, \mathrm{i}}$ depends on the mass transfer rate itself; $\dot{M}_{\mathrm{A}, \mathrm{i}}=\dot{M}_{\mathrm{A}, \mathrm{i}}\left(R_{10}, M_{\mathrm{wd}}, \dot{M}_{\mathrm{tr}}\right)$. The critical mass transfer rate is then given by a solution of

$\dot{M}_{\mathrm{tr}}=\dot{M}_{\mathrm{A}, \mathrm{i}}\left(R_{10}, M_{\mathrm{wd}}, \dot{M}_{\mathrm{tr}}\right)$,

(see also Stehle et al. 2001). Note that because the stream impact heating decreases with decreasing radius, the critical mass transfer rate is not necessarily defined by the vertical structure at the outer edge of the disc. In order to solve Eq. (7), we modify the approximation of the upper turning point of the S-curve by including impact heating. We obtain for the parameter range of SS Cyg:

$\log \left(\dot{M}_{\mathrm{A}, \mathrm{i}}\right)=\log \left(\dot{M}_{\mathrm{A}}\right)-0.039 \frac{Q_{\mathrm{i}}}{10^{10} \mathrm{erg} \mathrm{s}^{-1}}$.

Using this description, we solve Eq. (7) and obtain a series of solutions. The results can be approximated with the following power law:

$$
\begin{aligned}
\dot{M}_{\text {crit }, \mathrm{i}}= & 3.18 \times 10^{17} \mathrm{~g} \mathrm{~s}^{-1}\left(\frac{R_{10}}{5.83}\right)^{2.750} \\
& \times\left(\frac{\eta_{\mathrm{i}}}{1.0}\right)^{-0.267}\left(\frac{M_{1}}{1.19 M_{\odot}}\right)^{-0.287} .
\end{aligned}
$$

This leads to

$\dot{M}_{\text {crit }, \mathrm{i}}=(3.2 \pm 0.6) \times 10^{17} \mathrm{~g} \mathrm{~s}^{-1}$.

Figure 4 shows S-curves for the outer edge of the accretion disc assuming the above obtained mean mass transfer rate and considering the additional heating by the stream (Eq. (2)). The transition between $\alpha_{\text {hot }}=0.1$ and $\alpha_{\text {cold }}=0.02$ is assumed to be given by the following temperature dependence of $\alpha$ :

$$
\begin{aligned}
\log (\alpha)=\log \left(\alpha_{\text {cold }}\right)+ & {\left[\left(\log \left(\alpha_{\text {hot }}\right)-\log \left(\alpha_{\text {cold }}\right)\right]\right.} \\
\times & {\left[1+\left(\frac{2.5 \times 10^{4} \mathrm{~K}}{T_{\mathrm{c}}}\right)^{8}\right]^{-1}, }
\end{aligned}
$$

(see Hameury et al. 1998).

For the mean mass transfer rate we obtain $\overline{\dot{M}}_{\mathrm{tr}} \sim 2 \times$ $\dot{M}_{\mathrm{A}, \mathrm{i}}$ (Fig. 4), which would lead to steady disc accretion. Considering the uncertainties of the involved parameters, we find $\overline{\dot{M}}_{\mathrm{tr}} \gtrsim \dot{M}_{\text {crit }, \mathrm{i}}$.

\section{The onset of the cooling front}

The critical and the mean mass transfer rate derived in the previous section are sensitive to the uncertainties in the

${ }^{2}$ The weak dependence of $\dot{M}_{\mathrm{A}}$ on $\alpha_{\mathrm{h}}$ obtained by Hameury et al. (1998) and Schreiber \& Gänsicke (2001) is negligible in the context of this paper.

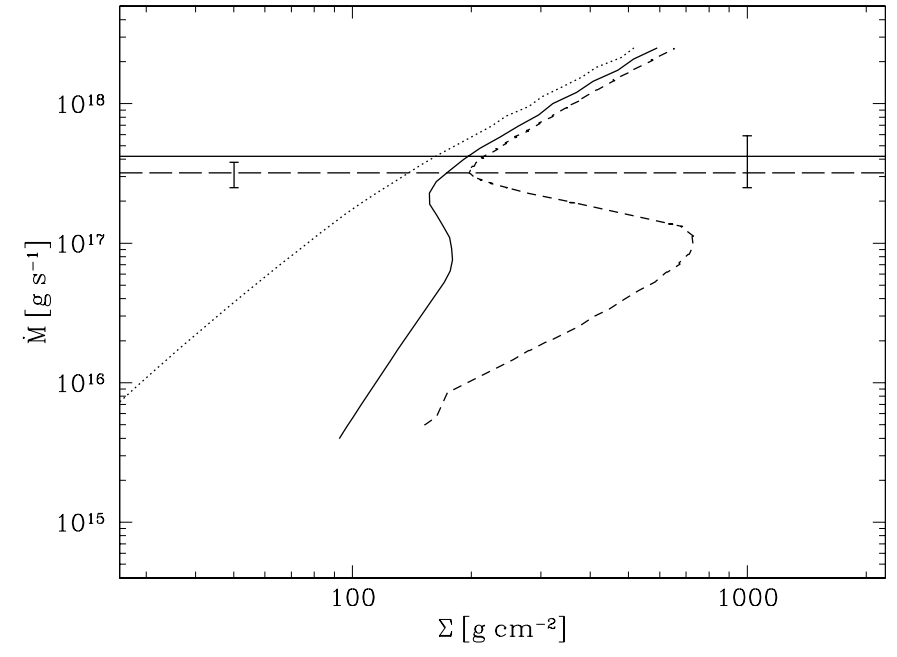

Fig. 4. S-curves for outer radii [in units of $10^{10} \mathrm{~cm}, R=$ $0.9 R_{\mathrm{L} 1}=5.83$ (dotted), $0.8 R_{\mathrm{L} 1}=5.18$ (solid), $0.7 R_{\mathrm{L} 1}=4.53$ (short dashed line)] using $\overline{\dot{M}}_{\mathrm{tr}}=4.2 \times 10^{17} \mathrm{~g} \mathrm{~s}^{-1}$ (solid horizontal line, see Eq. (5)). At the outer edge of the disc (dotted line) the instability is completely suppressed by the additional heating due to the stream $\left(\eta_{\mathrm{i}}=1.0\right)$. Moving inward, the additional heating decreases (see Eq. (2)) but the mean mass transfer rate (solid horizontal line) is still clearly above the value critical for stability. The critical accretion rate $\dot{M}_{\text {crit,i }}$ (see Eq. (10)) is represented by the dashed horizontal line. Notice, the S-curves are calculated with $\dot{M}_{\mathrm{tr}}=\overline{\dot{M}}_{\mathrm{tr}} \neq \dot{M}_{\mathrm{crit}, \mathrm{i}}$.

binary parameters and the assumed efficiency of stream impact heating $\eta_{i}$ as the additional heating can significantly reduce the critical mass transfer rate. Thus, while our results presented in Sect. 3 suggest that the DIM fails in describing the observations of SS Cyg, they do not allow to categorically exclude the validity of the DIM.

However, in this section we show in an independent way that the observed light curve of SS Cyg provides additional evidence for the failure of the DIM, independent from the details of stream impact heating or the uncertainties in the system parameters. For the mass accretion rate at the onset of the decline $\dot{M}_{\text {otd }}$ the DIM predicts an essentially lower value than required by the observations as, (a) the DIM predicts that the start of the cooling front at the outer edge of the disc defines the onset of the decline in the outburst light curve. The cooling front starts when the accretion rate of the quasi stationary disc is exactly equal to the critical rate, thus,

$\dot{M}_{\text {otd }, \text { DIM }}=\dot{M}_{\text {crit }, \mathrm{i}}=(3.2 \pm 0.6) \times 10^{17} \mathrm{~g} \mathrm{~s}^{-1} ;$

(b) the light curve of SS Cyg shows that there is no noticeable difference between the absolute magnitude at the onset of the decline and the outburst magnitude $m_{v}=$ $8.5 \pm 0.1$ (Fig. 1) and therefore, the observed light curve constrains the accretion rate at this point to be equal to the outburst accretion rate

$\dot{M}_{\text {otd }, \mathrm{obs}} \sim \dot{M}_{\mathrm{out}}=(3.62 \pm 1.47) \times 10^{18} \mathrm{~g} \mathrm{~s}^{-1}$.

The obtained huge discrepancy between the prediction of the DIM for $\dot{M}_{\text {otd }}$ (Eq. (12)) and the value required to 
explain the observed visual magnitude (Eq. (13)) clearly confirms the failure of the DIM.

It is worth noting that even if we completely neglect stream impact heating the DIM cannot explain the observations. Then, the critical mass transfer rate can be approximated by Eq. (6) and the accretion rate necessary to reach the visual flux during outburst somewhat increases:

$$
\begin{aligned}
& \dot{M}_{\text {otd }, \text { DIM }}=\dot{M}_{\text {crit }}=(7.6 \pm 2.0) \times 10^{17} \mathrm{~g} \mathrm{~s}^{-1}, \\
& \dot{M}_{\text {otd }, \text { obs }} \sim \dot{M}_{\text {out }}=(3.71 \pm 1.51) \times 10^{18} \mathrm{~g} \mathrm{~s}^{-1}, \\
& \dot{M}_{\text {tr }}=(4.3 \pm 1.8) \times 10^{17} \mathrm{~g} \mathrm{~s}^{-1} .
\end{aligned}
$$

Obviously, the problem for the DIM obtained in Sect. 3 vanishes if we neglect stream impact heating of the outer disc. Nevertheless, the DIM clearly fails as $\dot{M}_{\text {otd,obs }} \gg$ $\dot{M}_{\text {otd,DIM }}$.

\section{Discussion and conclusion}

Considering the "larger" distance of SS Cyg and the observed long term light curve, we calculated the mean mass transfer rate $\overline{\dot{M}}_{\text {tr }}$. Taking into account stream impact heating of the outer disc we find $\overline{\dot{M}_{\text {tr }}} \sim 2 \times \dot{M}_{\text {crit }}$ (Fig. 4) which contrasts with the predictions of the DIM: with such a high mean mass transfer rate, SS Cyg should not be a dwarf nova at all, but a novalike variable with a stationary hot accretion disc. This result is, however, sensitive to the assumed efficiencies of stream heating and disk irradiation and the uncertainties of the binary parameters, the inclination, and the distance. While this result on its own is not sufficient to proclaim a general failure of the DIM, an independent suggestion for a major problem with the DIM comes from comparing the accretion rate predicted by the DIM at the onset of the decline with the accretion rate required by the observed visual magnitude at this point (see Sect. 4). Hence, the conclusion from both these results must be: the current DIM cannot explain the observations of SS Cyg for a distance of $166 \pm 12 \mathrm{pc}$.

There are three possibilities that may resolve this problem: (a) the temperature dependence of $\alpha$, the key ingredient in the accretion disc limit cycle model, has to be modified, (b) increased mass transfer during the outburst plays an important rôle, or finally (c) the HST/FGS distance of $166 \mathrm{pc}$ and/or the assumed inclination are wrong.

Considering (a), it seems that we do not yet have an adequate understanding of the viscosity in accretion discs. The instability necessary to obtain the limit cycle behaviour appears mainly due to an artificial change of $\alpha$, given by Eq. (11), at the point where the small "natural", i.e. without a change of $\alpha$, ionisation instability has been found in the S-curves. There is no physical reason for this assumption and it might be the case that $\alpha$ starts to change at higher temperatures than previously assumed. This would lead to a higher value of the critical mass transfer rate. As such a modification would affect the dividing line between dwarf novae and nova likes, one should carefully consider the consequences for other dwarf nova before changing Eq. (11).

With respect to (b), we point out that another possibility to solve the problem obtained in Sects. $2-4$ is to assume that the mass transfer increases during the outbursts, e.g. due to irradiation of the secondary star. Assuming a mean mass transfer rate of $\overline{\dot{M}}_{\mathrm{tr} \text {,qui }}=1.5 \times 10^{17} \mathrm{~g} \mathrm{~s}^{-1}$ during quiescence, rise, and decay, the mean mass transfer rate during the outburst state should be

$$
\begin{aligned}
\overline{\dot{M}}_{\mathrm{tr}, \mathrm{out}} & \sim \frac{\dot{M}_{\mathrm{out}} t_{\mathrm{qs}}-\left(t_{\mathrm{qui}}+t_{\mathrm{ris}}+t_{\mathrm{dec}}\right) \overline{\dot{M}}_{\mathrm{tr}, \mathrm{qui}}}{t_{\mathrm{out}}-t_{\mathrm{ris}}-t_{\mathrm{dec}}} \\
& =2.4 \times 10^{18} \mathrm{~g} \mathrm{~s}^{-1},
\end{aligned}
$$

i.e. increased over the mean quiescent value by a factor of $\gtrsim 15$. In this scenario the onset of the decline in the light curve would represent decreasing mass transfer and not the start of the cooling front.

Finally, considering (c), we can only note that at least one of the additional CV parallaxes measured with HST, namely U Gem, agrees very well with previous distance estimates (Harrison et al. 1999). U Gem has a noticeably lower accretion rate than SS Cyg, therefore the distance estimated based on $K$ band magnitude and/or spectroscopy of the secondary star is much less affected by emission from the disc than in SSCyg. Thus, at least for U Gem an error in the HST/FGS parallax appears extremely unlikely. However, assuming $i=37^{\circ}$ and demanding $\dot{M}_{\text {out }} \sim 1.0 \times 10^{18} \mathrm{~g} \mathrm{~s}^{-1}$ for SS Cyg leads to $\overline{\dot{M}}_{\mathrm{tr}} \sim 1.2 \times 10^{17} \mathrm{~g} \mathrm{~s}^{-1}$ and requires $d \sim 117 \mathrm{pc}$ for agreement with the DIM.

Acknowledgements. This research has made use of the AFOEV database, operated at CDS, France. Special thanks are extended to Jean-Marie Hameury, Hans Ritter and Rick Hessman for interesting discussions at the CV-Conference in Göttingen (2001). We also wish to thank an anonymous referee for helpful comments. MRS thanks the Astronomy Department of the UCT for the warm hospitality during a nice and productive stay in March - May 2001 and the DAAD (PKZ:D/01/05718) for financial support. BTG thanks for support from the DLR under grant 50 OR 99036.

\section{References}

Beuermann, K. 2000, New Astron. Rev., 44, 93

Buat-Ménard, V., Hameury, J. M., \& Lasota, J. P. 2001, A\&A, 366,612

Buat-Ménard, V., Hameury, J. M., \& Lasota, J. P. 2001, A\&A, 369,925

Cannizzo, J. K. 1993a, ApJ, 419, 318

Cannizzo, J. K. 1993b, in Accretion disks in compact stellar objects, ed. J. Wheeler, Adv. Ser. Astrophys. Cosmol. 9 (Singapore: World Scientific), 6

Cannizzo, J. K. 1998, ApJ, 493, 426

Cannizzo, J. K., \& Mattei, J. A. 1992, ApJ, 401, 642

Cannizzo, J. K., \& Mattei, J. A. 1998, ApJ, 505, 344

Hameury, J., Menou, K., Dubus, G., Lasota, J., \& Hure, J. 1998, MNRAS, 298, 1048 
Hameury, J. M., Lasota, J. P., \& Dubus, G. 1999, MNRAS, Schreiber, M. R., Gänsicke, B. T., \& Hessman, F. V. 2000b, 303, 39 $\mathrm{A} \& \mathrm{~A}, 358,221$

Harrison, T. E., McNamara, B. J., Szkody, P., et al. 1999, ApJ, 515, L93

Harrop-Allin, M. K., \& Warner, B. 1996, MNRAS, 279, 219

Smak, J. 1984, Acta Astron., 34, 161

Stehle, R., King, A., \& Rudge, C. 2001, MNRAS, 323, 584

van Teeseling, A. 1997, A\&A, 324, L73

Ludwig, K., Meyer-Hofmeister, E., \& Ritter, H. 1994, A\&A, 290,473

van Teeseling, A., Reinsch, K., Pakull, M. W., \& Beuermann, K. 1998, A\&A, 338, 947

Paczynski, B., \& Schwarzenberg-Czerny, A. 1980, Acta Astron., 30, 127

Ritter, H. 1980, A\&A, 85, 362

Ritter, H., \& Kolb, U. 1998, A\&AS, 129, 83

Schreiber, M. R., \& Gänsicke, B. T. 2001, A\&A, 375, 937

Schreiber, M. R., \& Hessman, F. V. 1998, MNRAS, 301, 626

Schreiber, M. R., Gänsicke, B. T., \& Cannizzo, J. 2000a, A\&A,

Voloshina, I., \& Khruzina, T., 1997, in Accretion Phenomena and Related Outflows, ed. D. T. Wickramasinghe, F. Ferrario, \& G. Bicknell, ASP Conf. Ser., 121, 818

Warner, B. 1987, MNRAS, 227, 23

Warner, B. 1995, Cataclysmic Variable Stars (Cambridge: Cambridge University Press) 362, 268 